REGARDS

SUR LECONOMIE ALLEMANDE

BULLETIN ECONOMIQUE DU CIRAC
Regards sur l'économie allemande

Bulletin économique du CIRAC

$101 \mid 2011$

Varia

\title{
A plein régime
}

Isabelle Bourgeois

\section{OpenEdition}

Journals

Édition électronique

URL : http://journals.openedition.org/rea/4284

DOI : 10.4000/rea.4284

ISBN : 978-2-8218-0954-3

ISSN : 1965-0787

Éditeur

CIRAC

Édition imprimée

Date de publication : 10 juin 2011

Pagination : 3-4

ISSN : 1156-8992

Référence électronique

Isabelle Bourgeois, «A plein régime », Regards sur l'économie allemande [En ligne], 101 | juin 2011, mis en ligne le 19 septembre 2011, consulté le 15 septembre 2020. URL : http://journals.openedition.org/ rea/4284 


\section{A plein régime}

L'économie allemande a tourné le dos à la récession. Au premier trimestre, elle a enregistré une croissance de $1,5 \%$, après $0,4 \%$ seulement au cours du quatrième trimestre 2010 (Destatis). II ne s'agit pas là seulement d'une effervescence printanière au sortir d'un rude hiver. Car en comparaison avec le premier trimestre 2010, le PIB a connu une hausse de $4,9 \%$ en données CVS - un niveau jamais réitéré depuis le boom qui avait suivi I'Unité allemande. Après la chute brutale du PIB durant la crise, les activités ont repris continûment, trimestre après trimestre, et l'économie allemande a aujourd'hui renoué avec son niveau d'avant la crise. L'impact de la récession est « totalement surmonté » (Bundesbank, rapport de mai 2011).

La conjoncture est tirée par deux moteurs : l'export (un tiers de la croissance) et la demande intérieure (les deux tiers). La demande mondiale restant forte, le contexte monétaire favorable, le marché de l'emploi dynamique, le pouvoir d'achat en hausse et la politique budgétaire axée sur la consolidation, les experts s'accordent sur une croissance de l'ordre de $3 \%$ pour l'ensemble de l'année (les Instituts, dans leur rapport de printemps publié le 7 avril, avancent $+2,8 \%$ ). Après cette phase de rapide rattrapage, l'évolution de la conjoncture devrait se normaliser en 2012, et la croissance reprendre un rythme de croisière (+2,0\% selon les Instituts).

En mars, exportations et importations ont atteint un « volume record », comme se plaît à le souligner Destatis dans son communiqué du 9 mai : respectivement 98,3 milliards $€$ et 79,4 milliards $€$. La Bundesbank relativise quelque peu l'euphorie des statisticiens : cette évolution reflète surtout l'évolution des prix, particulièrement nette pour les importations. II n'empêche que la tendance reste bien orientée. Non seulement, la demande mondiale reste forte, mais les activités reprennent au sein de l'UE, où l'Allemagne a réalisé une large moitié de ses ventes (+ 16,0 \% par rapport à mars 2010) et les trois quarts de ses achats $(+21,1 \%)$. Dans la zone Euro, les exportations ont atteint 39,7 milliards $€(+14,2 \%)$, et les importations 36,1 milliards $€(+20,8 \%)$. Le volume des ventes à destination et des achats en provenance des pays tiers était respectivement de 39,5 milliards $€$ et de 28,1 milliards $€$. La catastrophe naturelle qui a frappé le Japon ne devrait guère avoir de répercussions sur la production en Allemagne ; en effet, les produits qu'elle importe sont essentiellement des biens d'investissement ou des biens de consommation haut de gamme (appareils et équipements électriques et optiques) s'accommodant d'une certaine flexibilité dans les délais d'approvisionnement, remarque la Bundesbank.

Depuis un an, la bonne tenue de l'investissement tire l'activité. L'an dernier, les investissements en biens d'équipement ont augmenté de $18 \%$, expliquent les Instituts, rappelant que si cette hausse vient en partie de ce que nombre de projets de renouvellement ajournés pendant la récession sont maintenant en cours de réalisation, elle a aussi pour origine la dynamique des commandes étrangères comme des exportations. En 2011, c'est l'extension des équipements qui devrait prendre le relais, le taux d'utilisation des capacités dépassant en ce moment la moyenne d'avant la récession. Au premier trimestre 2011, après avoir été ralenti par un hiver particulièrement rude, l'investissement dans la construction a connu un boom sous l'effet du besoin de rattrapage de l'industrie, mais aussi du fait d'une demande accrue des ménages, dopée par des conditions de crédit immobilier incitatives et des perspectives favorables sur le marché de l'emploi.

Le moral des ménages comme celui des entreprises est orienté au beau : l'indice ifo (entreprises) reste à un niveau élevé, les PME gardent confiance (KfW-ifo-Mittelstandsbarometer de mai), de même que les ménages. Certes, l'indice GfK a légèrement fléchi en mai, mais il reste à très haut niveau. Les consommateurs sont rassurés quant à l'évolution de l'emploi et à celle des salaires conventionnels : plusieurs conventions signées dans la chimie, le BTP, les télécommunications ou la fonction publique dans les Länder prévoient des hausses qui prennent effet dès le mois de mai, sans compter les primes décidées dans d'autres branches. Ajoutées au retour à la normale du nombre d'heures travaillées qui avait rehaussé les salaires au fil de l'année passée, ces perspectives seraient favorables à la consommation si le pouvoir d'achat n'était rogné par une hausse du coût de la vie qui atteignait 2,4\% en
Vers $3 \%$ de croissance en 2011

Bonne tenue du commerce mondial et intra-européen

L'investissement se maintient à haut niveau

Les ménages ont bon moral 
Moins de 3 millions de chômeurs attendus en 2011

Déficit : probablement moins de $2 \%$ en 2011 avril. L'inflation est due principalement à la flambée du coût des matières premières et encore plus des coûts de l'énergie : au premier trimestre, carburants et fuel domestique ont augmenté de $8 \%$ après une hausse de $7 \%$ à la fin 2010 (Bundesbank). Aujourd'hui, les consommateurs allemands ne redoutent plus le chômage ; leur premier souci est la stabilité des prix qu'ils craignent de voir mise à mal par l'explosion du prix de l'énergie et la crise de la dette au sein de la zone euro (GfK).

Car le marché de l'emploi reflète l'expansion des activités. Selon Destatis, en avril, le nombre d'actifs occupés atteignait 40,68 millions de personnes, soit un million de plus qu'en avril 2010. Quant au nombre de demandeurs d'emplois, il était tombé à 2,54 millions (soit un taux de 6,0 \% selon les critères de l'OIT) ; c'est dans le groupe des 15-24 ans qu'il a le plus fortement baissé (-25,4\%), portant le taux de chômage des jeunes à 7,9\%. Selon l'Agence pour l'emploi, le nombre de chômeurs indemnisés s'établissait en avril à 2,96 millions, soit un taux de 7,0\%. Quant au nombre de salariés en chômage partiel, il n'est plus que de 122000 en mars (586 000 de moins qu'un an auparavant), ce qui reflète la bonne santé de l'industrie. Le marché de l'emploi reste bien orienté, et sur l'ensemble de l'année, le nombre de chômeurs indemnisés devrait s'établir en moyenne à 2,93 millions seulement selon l'Institut IAB (Nuremberg) qui estime également le nombre de postes vacants à environ un million. Sachant qu'un grand nombre d'entreprises ne recourent pas aux services des 'pôles emploi' allemands, la demande de main-d'œuvre est en réalité nettement supérieure. La libre circulation de la main-d'œuvre au sein de l'UE depuis l'entrée en vigueur de la Directive Services au $1^{\text {er }}$ mai ou les politiques menées pour mieux concilier vies professionnelle et familiale ne suppléeront que partiellement à cette demande. Dans un rapport remis au gouvernement le 18 mai, le Conseil des Sages plaide dès lors pour un report de l'âge légal de départ à la retraite à 69 ans.

Les experts s'attendent à une « nette amélioration » (Bundesbank) de la situation des finances publiques. Le déficit, qui était remonté à 3,3\% du PIB en 2010, pourrait être ramené à moins de $2 \%$ cette année sous l'effet conjugué de plusieurs tendances : hausse de l'encours fiscal en période de forte croissance, recul des transferts $(0,5 \%$ du PIB en 2010) dédiés au sauvetage des établissements bancaires durant la crise (les banques commerciales sont dans l'ensemble tirées d'affaire), baisse des dépenses sociales du fait de la bonne tenue de l'activité comme de la fin des mesures exceptionnelles de soutien à l'emploi, et enfin réduction des dépenses publiques dans le cadre de la politique de consolidation du Bund qui a présenté le 13 avril son programme de stabilité budgétaire. Tout en saluant expressément le cap poursuivi, la Bundesbank ne rappelle pas moins que, «le niveau préoccupant de la dette exige que ce cap soit maintenu; d'autant plus que le contexte conjoncturel est favorable ». Les Instituts abondent dans le même sens et soulignent l'urgence d'une réforme de l'impôt sur le revenu. Car l'inflation gonfle les revenus imposables, ce qui accroît arithmétiquement le poids des prélèvements fiscaux "sans que ceux-ci reposent sur une productivité accrue ». Or pour préserver la compétitivité, il convient aussi d'étouffer toute tentation d'un 'second tour social'.

LES PRÉVISIONS DE CROISSANCE SONT CEPENDANT ENTACHÉES DE RISQUES. Le premier réside dans l'incertitude sur l'évolution des prix des matières premières et des produits pétroliers. Le deuxième, dans la résolution de la crise de la dette au sein de la zone euro dont l'impact sur la stabilité budgétaire allemande est imprévisible. Le troisième est interne à l'Allemagne : il tient aux répercussions sur l'économie de la sortie définitive du nucléaire à l'horizon 2022 décidée par le gouvernement fédéral (voir l'analyse dans ce numéro). Une envolée des prix de l'électricité n'est pas à exclure. Ni non plus une série de ruptures de l'approvisionnement en électricité. L'autorité de régulation des industries de réseaux (Bundesnetzagentur) est formelle : après l'arrêt de huit centrales en mars, " il ne sera pas possible d'assurer la sécurité du réseau électrique » à certaines périodes (communiqué du 27 mai). Dans une tribune libre publiée dans le quotidien BILD le 25 mars, l'ex-chancelier Helmut Kohl (CDU) mettait en garde le gouvernement fédéral contre toute sortie précipitée et isolée du nucléaire qui « viderait de sa substance le fondement même de [la] société industrielle qu'est l'Allemagne ». A moins que le gouvernement fédéral ne cherche au contraire à générer un sursaut de compétitivité industrielle sur la base de son avancée technologique ...

I. Bourgeois (01-06-2011) 